\title{
Alcohol consumption is associated with the severity and outcome of acute liver injury/failure
}

\author{
Puneet Puri ${ }^{1,2}$ (D) | William M. Lee ${ }^{3}$ (D) | Robert J. Fontana ${ }^{4}$ | Nak-Kyeong Kim ${ }^{5}$ | \\ Valerie Durkalski $^{6}$ | Brendan M. McGuire ${ }^{7}$ | Iris Liou ${ }^{8}$ | Carla Pezzia9 | \\ R. Todd Stravitz ${ }^{2}$ for the Acute Liver Failure Study Group
}

${ }^{1} \mathrm{GI}$ Section, Hunter Holmes McGuire Department of Veterans Affairs Medical Center, Richmond, VA, USA

${ }^{2}$ Hume-Lee Transplant Center of Virginia Commonwealth University, Richmond, VA, USA

${ }^{3}$ University of Texas, Southwestern Medical Center, Dallas, TX, USA

${ }^{4}$ University of Michigan, Ann Arbor, MI, USA

${ }^{5}$ Department of Biostatistics, Virginia Commonwealth University, Richmond, VA, USA

${ }^{6}$ Medical University of South Carolina, Charleston, SC, USA

${ }^{7}$ University of Alabama, Birmingham, AL, USA

${ }^{8}$ Division of Gastroenterology, Department of Medicine, University of Washington, Seattle, WA, USA

${ }^{9}$ Department of Human and Social Sciences, University of Dallas, Dallas, TX, USA

\section{Correspondence}

Richard Todd Stravitz, M.D., Professor of Medicine, Medical Director of Liver Transplantation, Hume-Lee Transplant Center of Virginia Commonwealth University, PO Box 980341, Richmond, VA 23298-0341, USA.

Email: Richard.Stravitz@VCUHealth.ORG

\section{Funding information}

The Acute Liver Failure Study Group receives funding from the National Institutes of Health/National Institute of Diabetes, Digestive and Kidney Diseases Grant U-01 58369. Support to Puneet Puri by National Institute on Alcohol Abuse and Alcoholism, Grant K23AA021179.

Handling editor: Dominique Thabut

\begin{abstract}
Background \& Aims: Non-medical factors which contribute to the severity of acute liver failure (ALF) remain poorly defined. The association of alcohol consumption on the severity of presentation and outcome were determined in patients with ALF and acute liver injury (ALI) in a large, multicentre registry.

Methods: Alcohol consumption during the 6 months prior to study entry was analysed in 1170 patients enrolled in the ALF Study Group Registry. Consumption was categorized as none/minimal ( $<3$ alcoholic beverages/week) or at least moderate ( $\geq 3$ / week). Clinical characteristics, the severity of liver injury at presentation (ALI or ALF) and outcome were compared.

Results: In patients with acetaminophen (APAP) overdose, at least moderate alcohol consumption was associated with higher peak aminotransferases, bilirubin, creatinine and INR on admission, compared to no/minimal consumption. In patients with non-APAP ALI/ALF, at least moderate alcohol consumption was associated with higher peak aminotransferases and creatinine. In APAP, non-APAP or all aetiologies, at least moderate alcohol consumption was associated with a $75 \%, 89 \%$ and $82 \%$ higher odds, respectively, of presenting as ALF rather than ALI (all $P<.005$ ). At least moderate alcohol consumption increased the odds of death by $45 \%(P=.01)$ across all aetiologies. In multivariate analysis, older age, non-Caucasian race, peak INR, peak bilirubin and at least moderate alcohol consumption were significantly associated with death. Finally, in Kaplan-Meier analysis of patients with all aetiologies, at least moderate alcohol consumption was associated with decreased time-dependent survival $(P=.002)$.
\end{abstract}

Conclusion: Alcohol consumption adversely affects the presentation and outcome of both APAP- and non-APAP-induced ALI/ALF.

\section{KEYWORDS}

acetaminophen, acute liver failure, alcohol, coagulopathy, ethanol, hepatic encephalopathy 


\section{1 | INTRODUCTION}

Acute liver failure (ALF) is a clinical syndrome consisting of a primary liver injury followed by secondary multi-organ system failure (MOSF), culminating in death or the need for liver transplantation (LT) in more than half of affected individuals. ${ }^{1}$ The aetiology of the primary liver injury may be a toxin or drug, or less commonly, a viral, vascular or metabolic injury. In the United States and parts of Western Europe, acetaminophen (APAP) is the most common toxin incriminated in ALF, accounting for nearly half of cases. ${ }^{1,2}$ Clinical features of patients with ALI/ALF which increase the susceptibility to, or severity of, primary liver injury have not been well-defined.

Although alcohol consumption has been reported to decrease the threshold of APAP hepatotoxicity, ${ }^{3}$ the interaction is complex, and studies have not universally agreed that alcohol increases the hepatotoxicity of APAP. In experimental animals (highly controlled conditions), alcohol increases hepatotoxicity of APAP by induction of specific cytochrome P-450's (both P-450 3a and $1 \mathrm{a}^{4}$ ), thereby shunting more APAP into the metabolic pathway to produce the toxic metabolite, $\mathrm{N}$-acetyl-p-benzoquinoneimine (NAPQI) and away from the non-toxic production of glucuronides or sulphates. ${ }^{5}$ In humans (highly variable conditions), the timing, chronicity and quantity of both the alcohol and the APAP exposure determine the degree of liver injury. Other poorly defined cofactors modulate this interaction, such as fasting and/or the nutritional state of the patient, which determine glutathione reserves. ${ }^{6,7} \mathrm{~A}$ less well-documented interaction between alcohol and APAP-induced liver injury has been hypothesized to occur with the co-ingestion of alcohol and APAP, in which experimental animals and humans appear to be more resistant to liver injury because of competition of alcohol and APAP for metabolism by the same cytochrome P450's, resulting in decreased NAPQI formation.,

The independent contribution of regular alcohol consumption (not abuse) to the severity of presentation and outcome of ALI/ALF has not been well-established in patients with APAP overdose partly because study populations have not been large enough to account for the heterogeneity of alcohol and APAP ingestion. Furthermore, the association of alcohol consumption on the severity on ALI/ALF of non-APAP-induced liver injury has not been explored. The ALF Study Group Registry has prospectively collected alcohol consumption data on participants with ALF and ALI (a less severe liver injury without hepatic encephalopathy) since its inception in 1998. The work presented herein attempts to quantify the association of alcohol consumption on the severity of liver injury in patients with ALI/ALF of diverse aetiologies, and to determine whether alcohol consumption adversely affects the clinical course of patients with non-APAP liver injury.

\section{2 | METHODS}

\section{1 | Study subjects}

Patient accrual followed the algorithm in Figure 1. Between 1998 and 2015, 2670 patients with ALI and ALF have been enrolled in

\section{Keypoints}

1. Acute liver failure is frequently a life-threatening condition with high mortality.

2. Many etiologies of ALF exist, most commonly acetaminophen in Western countries.

3. This study suggests that even moderate alcohol consumption increases the severity of presentation and clinical course of liver injury in acetaminophen as well as non-acetaminophen ALF.

the ALF Study Group Registry wherein detailed data and daily biosamples are collected over 7 days. All study patients met criteria for $A L F$ or $A L I$ as defined previously; in order to ensure patients with $A L I$ had severe liver dysfunction, we have previously defined ALI as INR $\geq 2.0^{1,10}$. Patients were excluded if they had underlying liver disease, including a previous history of alcohol-induced liver injury. Consent was provided by the patient if they exhibited no evidence of hepatic encephalopathy (ALI), and the patients' legal next-of-kin, if they had any signs of encephalopathy (ALF). Institutional Review Boards of each participating institution have approved the ALF Study Group Registry.

Of the 2670 patients with ALI/ALF who were screened, data on alcohol use were available in 1170 subjects (Figure 1). Of the 1500 excluded subjects, alcohol use data were missing on 1202 and 21day outcome on the remaining subjects. The ALF Study Group began recruiting patients with ALI in 2010, and data regarding alcohol consumption were less completely collected before this time. As a consequence, only $4.6 \%$ of the patients excluded from the study had ALI compared to $26 \%$ of the included group $(P<.0001)$, and the excluded group was, therefore, more likely to have poor outcomes (death or LT) than the included group $(P<.0001$; data not shown).

The aetiology of ALI/ALF was APAP in 591 patients (51\%), and nonAPAP in 579 patients (49\%). Among the non-APAP aetiologies, autoimmune hepatitis was diagnosed in $8 \%$, drug-induced in $7.6 \%$, hepatitis $\mathrm{B}$ in $7.3 \%$, shock/ischaemia in $6.6 \%$ and other minor aetiologies in $5.7 \%$ (pregnancy-associated liver failure, Budd-Chiari Syndrome, hepatitis $\mathrm{A}, \mathrm{C}$ and $\mathrm{E}$, other viruses, mushroom poisoning and Wilson disease; each $1 \%$ or less). Indeterminate aetiology was diagnosed after extensive but unrevealing evaluation in $10 \%$. Spontaneous (transplant-free) survival at day 21 was reported in 703 (60\%), overall survival in 895 (77\%), LT in 177 (15\%) and death in 275 (24\%).

\section{2 | Definitions of alcohol consumption}

On the day of admission to the Registry, sites were asked to record the patient's 'usual intake of alcohol during the last 6 months'. One 'drink' of alcohol was defined as $12 \mathrm{oz}$. (1 can or bottle) of beer, $5 \mathrm{oz}$. (1 glass) of wine, $1.5 \mathrm{oz}$. of hard liquor or $12 \mathrm{oz}$. (1 bottle) of wine cooler. The sum of the number of drinks per week was then recorded 


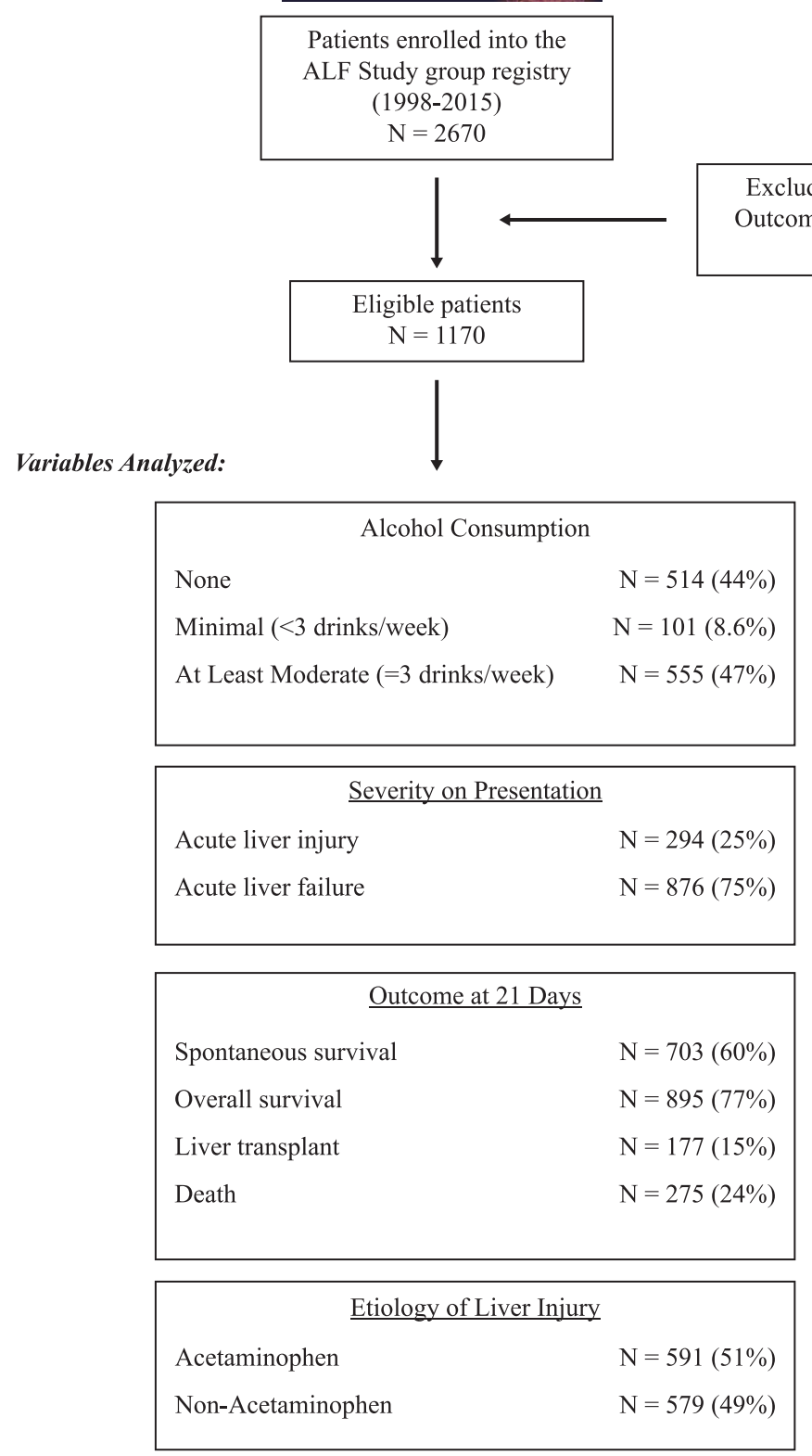

as none (<1 drink/week), 1, 2, 3-6, 7-14 and >14 drinks/week. The number of weeks of alcohol consumption during the last 6 months was also recorded. Patients were distributed into groups according to alcohol consumption as follows: none ( $<1$ drink/week), minimal $(<3$ drinks/week) or at least moderate ( $\geq 3$ drinks/week). The no alcohol and minimal alcohol groups were combined as they were shown to be similar in demographics, laboratories and outcomes (data not shown).

\section{3 | Statistical analyses}

The association of alcohol consumption on the severity and outcome of liver injury was analysed separately in patients with APAPinduced and non-APAP-induced ALI/ALF. The effects of alcohol consumption on the severity of presentation and outcomes of ALI/ ALF were, therefore, analysed comparing patients who consumed no/minimal vs those who consumed at least moderate alcohol. The
FIGURE 1 Study overview
Excluded for Alcohol or come Status Unknown $\mathrm{N}=1500$ statistical methods included descriptive statistics, multiple logistic regression, Kaplan-Meier curves and log-rank test.

\section{3 | RESULTS}

\section{1 | Patient characteristics according to alcohol consumption in the 6 months prior to presentation for ALI/ALF}

The clinical characteristics of study participants are displayed in Table 1 (comparing demographic and peak laboratory values) and Table S1 (comparing admission laboratory values) dichotomized by the aetiology of liver injury, APAP-induced and non-APAP-induced. Overall, 615 patients (53\%) consumed no/minimal amounts of alcohol in the 6 months prior to admission, and 555 (47\%) consumed at least moderate amounts. The number of subjects who reported 


\begin{tabular}{|c|c|c|c|}
\hline \multirow[b]{2}{*}{ Clinical feature } & \multicolumn{2}{|c|}{ Alcohol consumption } & \\
\hline & $\begin{array}{l}\text { None/minimal } \\
\mathrm{N}=615\end{array}$ & $\begin{array}{l}\text { At least moderate } \\
\mathrm{N}=555\end{array}$ & \\
\hline $\begin{array}{l}\text { Acetaminophen aetiolo } \\
(\mathrm{N}=591)\end{array}$ & $N=243$ & $N=348$ & $P^{*}$ \\
\hline Age (y) & $37.6 \pm 14.1$ & $36.9 \pm 11.7$ & 0.50 \\
\hline Gender (\% female) & 78.2 & 63.8 & 0.0002 \\
\hline Race (\% Caucasian) & 83.5 & 85.1 & 0.61 \\
\hline Peak AST (IU/L) & 5279 (2606-8853) & 8000 (3857-13785) & $<0.0001$ \\
\hline Peak ALT (IU/L) & 4667 (2003-7432) & $5373(2593-8887)$ & 0.018 \\
\hline Peak bilirubin (mg/dl) & $3.6(2.2-5.0)$ & $5.0(3.2-7.4)$ & $<0.0001$ \\
\hline Peak creatinine (mg/dl) & $1.5(0.9-2.7)$ & $2.2(1.1-3.6)$ & $<0.0001$ \\
\hline Peak INR & $3.8(2.6-5.8)$ & $4.2(2.8-6.2)$ & 0.16 \\
\hline $\begin{array}{l}\text { Non-acetaminophen ae } \\
(\mathrm{N}=579)\end{array}$ & $N=372$ & $N=207$ & \\
\hline Age (y) & $45.4 \pm 16.2$ & $44.1 \pm 15.1$ & 0.34 \\
\hline Gender (\% female) & 63.7 & 48.8 & 0.0005 \\
\hline Race (\% Caucasian) & 61.0 & 76.8 & 0.0001 \\
\hline Peak AST (IU/L) & $1776(752-3819)$ & 2184 (938-5732) & 0.04 \\
\hline Peak ALT (IU/L) & $1490(635-3195)$ & 1953 (869-4755) & 0.017 \\
\hline Peak bilirubin (mg/dl) & $15.6(6.2-23.6)$ & $12.8(5.9-23.2)$ & 0.50 \\
\hline Peak creatinine (mg/dl) & $1.2(0.8-2.4)$ & $1.6(0.9-3.2)$ & 0.007 \\
\hline Peak INR & $2.8(2.0-4.4)$ & $3.0(2.2-4.8)$ & 0.63 \\
\hline
\end{tabular}

TABLE 1 Clinical and peak laboratory features of study population according to alcohol consumption during the six months prior to admission for ALI/ALF

Note: Peak values were the highest recorded during the first 7 days of admission (prior to liver transplantation, if applicable).

Normal data are expressed as mean $\pm \mathrm{SD}$; non-normal data are expressed as median (IQR 25\%-75\%).

${ }^{*}$ As defined in Methods, data from patients with no/minimal alcohol consumption are compared to those with at least moderate alcohol consumption.

weekly alcohol consumption of 3-6 drinks ( $n=129)$, 7-14 drinks $(n=124),>14$ drinks $(n=302)$ represented the group with at least moderate amounts of alcohol consumption.

The clinical characteristics of study participants differed significantly between the dichotomized aetiologies of ALI/ALF. Patients with APAP-induced liver injury were younger and more predominantly female compared to patients with non-APAP-induced ALI/ $\operatorname{ALF}(37.3 \pm 12.7$ vs $45.0 \pm 15.8$ years and $70 \%$ vs $58 \%$ respectively; both $P<.0001)$. As is characteristic of APAP-induced liver injury, aminotransferases were three- to four-fold higher, and total bilirubin levels were $~ 75 \%$ lower, than those with non-APAP-induced ALI/ ALF (both $P<.001$ ). Admission serum creatinine was also significantly higher in patients with APAP vs non-APAP aetiology (1.5 [0.83.1] vs 1.25 [0.8-2.4] mg/dl respectively; $P=.01$, data not shown).

Of those with APAP-induced ALI/ALF, female patients were more commonly observed in the group who consumed no/minimal amounts of alcohol compared to the group who consumed at least moderate amounts (78.2\% vs 63.8\%, $P=.0002$ ) (Table 1). Peak AST and ALT levels were significantly higher in patients who consumed at least moderate alcohol compared to those who consumed no/minimal quantities (8000 vs 5279 IU/L [P <.0001] and 5373 vs $4667 \mathrm{IU} / \mathrm{L}$
$[P=.018]$ respectively). Patients who consumed at least moderate quantities of alcohol also developed significantly higher peak total bilirubin $(P<.0001)$ and creatinine $(P<.0001)$, than patients who consumed no/minimal amounts of alcohol. Similar trends were observed in laboratory values on admission to the Registry (Table S1).

Of patients with non-APAP-induced ALI/ALF (Table 1), more females were again observed in the no/minimal alcohol group $(P=.0005)$, and Caucasians were more frequently observed in the at least moderate alcohol group $(P=.0001)$. Peak AST and ALT were also higher in the group who consumed at least moderate alcohol than those in the none/minimal group (2184 vs $1776 \mathrm{IU} / \mathrm{L}[P=.04]$ and 1953 vs $1490 \mathrm{IU} / \mathrm{L}[P=.017]$ respectively), as was the peak serum creatinine (1.6 [0.9-3.2] vs 1.2 [0.8-2.4] mg/dl respectively; $P=.007)$. Similar trends were observed in laboratory values on admission (Table S1).

\subsection{Association of alcohol consumption on the presentation and outcome of ALI/ALF}

The association of alcohol consumption on the severity of liver injury on presentation and the outcome at 21 days are depicted in Table 2. 
TABLE 2 Effects of alcohol consumption on the presentation and outcome of ALI/ALF

\begin{tabular}{|lllll}
$\begin{array}{l}\text { Presentation } \\
\text { or outcome }\end{array}$ & $\begin{array}{l}\text { Aetiology of } \\
\text { ALI/ALF }\end{array}$ & $\begin{array}{l}\text { Odds } \\
\text { ratio }\end{array}$ & $95 \% \mathrm{Cl}$ & $\boldsymbol{P}$ \\
\hline ALF vs ALI & APAP & 1.75 & $1.20-2.54$ & 0.003 \\
& Non-APAP & 1.89 & $1.24-2.92$ & 0.002 \\
& All aetiologies & 1.82 & $1.38-2.42$ & $<0.0001$ \\
\hline Death/LT & APAP & 1.28 & $0.87-1.89$ & 0.19 \\
vs SS & Non-APAP & 1.31 & $0.93-1.85$ & 0.12 \\
& All aetiologies & 1.30 & $1.01-1.68$ & 0.046 \\
\hline Death vs alive & APAP & 1.77 & $1.13-2.77$ & 0.01 \\
& Non-APAP & 1.18 & $0.81-1.72$ & 0.37 \\
& All aetiologies & 1.45 & $1.09-1.94$ & 0.01 \\
\hline
\end{tabular}

Abbreviations: APAP, acetaminophen; $\mathrm{Cl}$, confidence interval; $\mathrm{LT}$, liver transplantation; SS, spontaneous (transplant-free) survival.

Odds ratio and $95 \%$ confidence interval $(\mathrm{Cl})$ represents the increased risk of the more severe presentation or worse outcome in patients who consumed at least moderate alcohol compared to those who consumed no or minimal alcohol in the 6 months prior to presenting with ALI or ALF, as defined in Methods.

For the severity of presentation, at least moderate alcohol consumption was associated with an increased odds of ALF vs ALI of $75 \%$ in patients with APAP aetiology (OR 1.75 [1.20-2.54]; $P=.003$ ), of $89 \%$ in patients with non-APAP aetiology (OR 1.89 [1.24-2.92]; $P=.002$ ), and of $82 \%$ in patients with all aetiologies (OR 1.82 [1.38-2.42]; $P<.0001)$, compared to patients with no/minimal alcohol consumption in the 6 months prior to admission. Alcohol consumption was also associated with adverse outcomes of ALI/ALF. In patients with ALF/ALI of all aetiologies, at least moderate alcohol consumption increased the odds of death or LT by $30 \%$ compared to those who consumed no/minimal quantities (OR 1.30 [1.01-1.68]; $P=.046$ ). One hundred thirty patients in no/minimal category and 47 patients with at least moderate alcohol consumption underwent LT. Among the APAP-induced ALI/ALF, 15 patients in no/minimal category and seven patients with at least moderate amounts of alcohol underwent LT. Overall survival was also adversely affected by alcohol consumption in patients with APAP-induced ALI/ALF, with a $77 \%$ increase in the odds of death in patients who consumed at least moderate amounts compared to no/minimal amounts (OR 1.77 [1.13-2.77]; $P=.01$ ), and an increase of $45 \%$ in all patients regardless of aetiology (OR 1.45 [1.09-1.94]; $P=.01$ ).

In multivariate analysis, older age, non-Caucasian race, peak INR, peak bilirubin and at least moderate alcohol consumption were significantly associated with death at 21 days after enrolment in the ALF Study Group Registry (Table 3). Substitution of admission laboratory values into the multivariate analysis yielded similar results, except that higher AST, ALT and creatinine were also associated with death, while at least moderate alcohol consumption was not $(P=$.14). Finally, Kaplan-Meier curves showed a significant reduction in 21-day survival in patients with a history of at least moderate alcohol consumption compared to no/minimal alcohol consumption (Figure 2; $P=.002$ by log-rank test).
TAB LE 3 Risk of death at 21 days after admission for ALI/ALF by multivariate analysis based upon peak laboratories (day 1-7)

\begin{tabular}{|c|c|c|c|c|}
\hline \multirow[b]{2}{*}{ Clinical feature } & \multicolumn{2}{|c|}{ Univariate analysis } & \multicolumn{2}{|c|}{$\begin{array}{l}\text { Multivariate } \\
\text { analysis }\end{array}$} \\
\hline & OR & $P$ & OR & $P$ \\
\hline Age & 1.03 & $<0.0001$ & 1.04 & $<0.0001$ \\
\hline Female gender & 0.79 & 0.10 & 0.77 & 0.14 \\
\hline Caucasian race & 0.65 & 0.008 & 0.65 & 0.026 \\
\hline Peak AST & 1.00 & 0.17 & 1.00 & 0.07 \\
\hline Peak ALT & 0.99 & 0.043 & 0.99 & 0.26 \\
\hline Peak bilirubin & 1.02 & $<0.0001$ & 1.01 & 0.007 \\
\hline Peak creatinine & 1.00 & 0.63 & 0.99 & 0.64 \\
\hline Peak INR & 1.01 & 0.07 & 1.02 & 0.048 \\
\hline APAP aetiology & 0.55 & $<0.0001$ & 0.99 & 0.96 \\
\hline $\begin{array}{l}\text { At least moderate } \\
\text { alcohol consumption }\end{array}$ & 1.22 & 0.15 & 1.44 & 0.039 \\
\hline
\end{tabular}

Abbreviation: OR, odds ratio of death (units OR for continuous variables [age, INR, AST, ALT, bilirubin and creatinine] measuring per unit change in regressor).

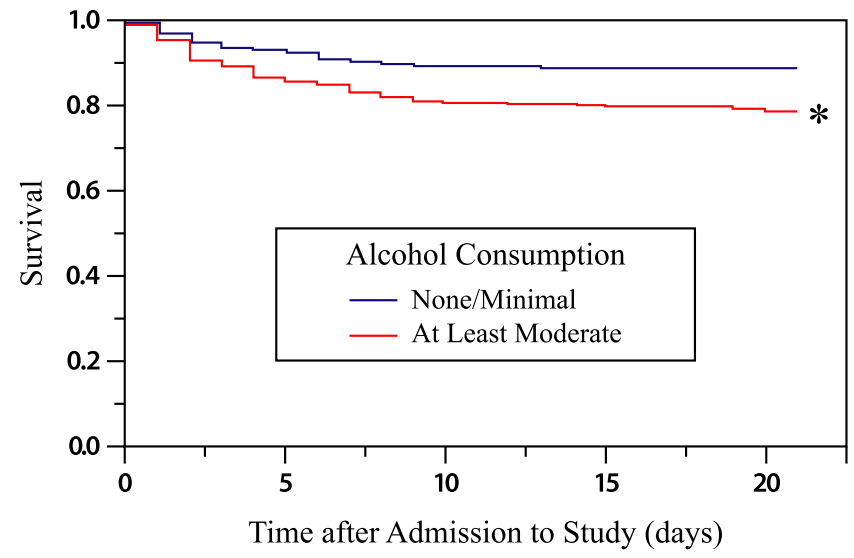

FIGURE 2 Kaplan-Meier survival of patients with ALI/ALF (all aetiologies) according to alcohol consumption, At Least Moderate vs None/Minimal ( ${ }^{*}$ Log-Rank test $P=.002$ )

\section{DISCUSSION}

The data presented suggest that alcohol consumption adversely affects the severity of clinical presentation and outcome of both APAP- and non-APAP-induced ALF/ALI. Patients with at least moderate alcohol consumption were 75\% (APAP) and $89 \%$ (nonAPAP) more likely to present with ALF vs ALI, compared to patients with no/minimal alcohol consumption in the 6 months prior to presentation with liver injury. A similar adverse effect on outcome was observed for all aetiologies in patients with at least moderate alcohol consumption compared to no/minimal consumption, with a $30 \%$ increase in death or LT and $45 \%$ increase in overall death. We also found that alcohol consumption increased the risk of overall death exclusive of the risk on LT. In fact, alcohol consumption did not significantly affect LT rates, suggesting 
that alcohol consumption did not bias our results by affecting access to transplant (the transplant rate in the no/minimal group was $13.4 \%$ vs $17.7 \%$ in the at least moderate group; $P=.08$ ). This observation also supports a general consensus by transplant clinicians at our study sites that few patients in our population abused alcohol.

Although an adverse effect of chronic alcohol abuse on the outcome of APAP overdose has been previously observed, our data are primarily based upon consumers of alcohol, not those who would be considered having a risk of "alcohol use disorder" as defined by the National Institute of Alcohol Abuse and Alcoholism (NIAAA); fewer than half of patients who consumed any alcohol in the 6 months prior to presentation would meet criteria for possible alcohol use disorder (data not shown). Thus, our findings raise concern that alcohol consumption even in modest amounts may be a co-factor in the clinical course of liver injury regardless of aetiology. We had no way to exclude the possibility of a role of subclinical alcoholic liver disease in the acute injury experienced by our cohort of study patients. However, overt alcoholic liver disease and a history of any chronic liver disease were exclusion criteria for entry into the Registry. Therefore, since the majority of our patients would not be considered to have abused alcohol, we believe pre-existing, clinically significant alcoholic liver disease was an unlikely explanation for the more severe presentation and increased poor outcomes in our study population with ALI/ALF.

Previous studies have generally suggested that chronic alcohol consumption does not increase APAP hepatotoxicity; the association was termed "at best, ambiguous" by the authors of the largest study prior to the present. ${ }^{9}$ In this cohort of 645 patients with single-dose APAP overdose, however, chronic alcohol abuse was an independent risk factor for hepatic encephalopathy and mortality. Many less rigorously-collected databases have not revealed alcohol consumption as a risk factor for mortality after APAP overdose. ${ }^{11}$ In an analysis from the King's College, ${ }^{12}$ overall survival of patients with a history of heavy alcohol consumption was similar to non-drinkers. In a small subset of our ALF Study Group Registry patients enrolled between 1998 and $2003,{ }^{13}$ alcohol use was not associated with adverse outcome (based upon 273 patients; $P=.21$ ) and alcohol abuse was actually associated with higher transplant-free survival (based upon 196 patients; $P=.04$ ). However, those with a history of alcohol abuse presented with lower grade hepatic encephalopathy, suggesting that they were less acutely ill. The fact that chronic heavy alcohol consumption increases mortality inconsistently after APAP overdose may lie in poorly defined features of the APAP ingestion, which differ according to single ingestions with suicidal intent or the 'therapeutic misadventure' unintentional overdose, or an association of heavy alcohol consumption with larger doses of ingested APAP. ${ }^{12,14}$ There are other historical features of these complex ingestions which have proved almost impossible to collect accurately, such as the precise dose and timing of APAP ingestion, whether there were chronic, repeated exposures to APAP such as in bundled narcotic abuse, and fasting and nutritional state before the APAP ingestion.
In contrast to chronic alcohol consumption, acute alcohol exposure has been postulated to protect against APAP hepatotoxicity ${ }^{8,9}$; thus, the timing of the APAP ingestion after ethanol consumption appears to be a determinant of the odds of enhanced liver injury, and could have confounded our data. Although we did not collect the time of most recent alcohol consumption before APAP ingestion, the benefit of recent alcohol ingestion on APAP overdose appears to be short-lived. In a human study, APAP ingestion eight hours after an ethanol infusion was associated with a $22 \%$ increase in NAPQI in blood and urine compared to APAP ingestion after a glucose infusion, ${ }^{15}$ suggesting that even a near-contemporaneous exposure to alcohol may not diminish APAP hepatotoxicity. Although a protective effect of recent alcohol consumption on APAP hepatotoxicity has been shown in laboratory animals, ${ }^{8,16}$ a possible benefit has been difficult to observe even in carefully executed human studies in which the investigators recorded instances of co-ingestion of APAP and alcohol. Specifically, Schmidt, et $\mathrm{al}^{9}$ found a protective effect of acute alcohol ingestion (defined as alcohol consumption as part of the APAP intoxication) on APAP hepatotoxicity only in the subset of patients with a history chronic alcohol consumption; acute alcohol consumption was not protective in non-alcoholic patients.

Previous studies have identified other patient characteristics which appear to potentiate the toxicity of APAP on the liver. Older age has been shown to increase the mortality of APAP and nonAPAP ALF., 17-19 Specific medications which modulate tissue injury and repair, such as statins and non-steroidal inflammatory drugs, have been associated with decreased hepatotoxicity from APAP overdose. ${ }^{20,21}$ Fasting and malnutrition increases APAP hepatotoxicity by depleting glutathione stores $^{6}$; somewhat paradoxically, obesity and previous bariatric surgery have also been incriminated. ${ }^{22,23}$ We found that, in addition to older age and at least moderate alcohol consumption, non-Caucasian race was an independent predictor of higher overall 21-day mortality, which could reflect differences in access to medical care or liver transplantation, or genetic differences in the metabolism of APAP.

Consistent with data regarding presentation and outcome, alcohol consumption also increased biochemical evidence of liver injury, depending upon whether peak (Table 1) or admission (Table S1) laboratory values were examined. For peak labs, AST, ALT and creatinine were higher for both APAP and non-APAP aetiologies in patients who consumed at least moderate alcohol compared to those who consumed no/minimal alcohol, as was peak bilirubin in those with APAP overdose. Similar trends were observed regarding admission laboratories, where bilirubin and INR were significantly higher in those with APAP overdose who consumed at least moderate alcohol, but differences were less striking in those with nonAPAP aetiology. The finding that alcohol may increase liver injury in non-APAP ALI/ALF has not been previously reported. As shown in Table 3 and Table S2, many features (age, race, laboratory data, aetiology and alcohol consumption) contribute to the risk of death after ALI/ALF depending upon which laboratories are entered into the multivariate model. The finding that alcohol consumption drops 
out of the model if admission rather than peak laboratories are used reflects the complex interaction of these variables, and may be the reason that previous studies have not consistently found an adverse effect of alcohol consumption on the outcome of ALF.

We acknowledge limitations of our study. Most importantly, granular details regarding ethanol consumption history were not available, in particular, the timing of the last alcohol ingestion. Similarly, granular details regarding the dose and timing of one or more APAP ingestions are also exceedingly difficult to obtain in this critically ill population. The reliability and accuracy of the semi-quantitative assessment of alcohol consumption by patients with altered mentation may be questioned. The timing of last meal, as an indicator of fasting state, was also not collected. The large number of patients excluded for missing critical data (alcohol consumption and/or outcome) is also a significant limitation, although after comparing the included and excluded populations (data not shown), it is not clear how this affected our results.

In conclusion, alcohol consumption, including a modest consumption, may increase the risk of presenting with hepatic encephalopathy (ALF) rather than ALI for patients with APAP- and non-APAP-induced liver injury. At least moderate alcohol consumption may increase the risk of death in patients with ALF/ALI of all aetiologies, although the interplay between risk variables is complex. Further study to explain the mechanism by which alcohol consumption increases the liver injury after a non-APAP insult should be undertaken to better prove the association.

\section{ACKNOWLEDGEMENTS}

The authors wish to gratefully acknowledge the support of our NIH/ NIDDK Project Officers for the ALF Study Group, Drs. Edward Doo and Averell Sherker.

\section{CONFLICT OF INTEREST}

The authors have no disclosures which would represent a conflict of interest in any regard to the content of this work. We have no competing interests to report.

\section{AUTHOR CONTRIBUTIONS}

Puneet Puri, MD: Planning/conducting study, Analysis of the data, Drafting manuscript. William M. Lee, MD: Conducting study, Interpreting data, Drafting manuscript. Robert J. Fontana, MD: Conducting study, Interpreting data, Drafting manuscript. N-K Kim, PhD: Statistical analysis. Valerie Durkalski, PhD: Conducting study, Interpreting data. Brendan McGuire, MD: Conducting study, Interpreting data, Drafting manuscript. Iris Liou, MD: Conducting study, Interpreting data, Drafting manuscript. Carla Pezzia, PhD: Interpreting data, Drafting manuscript. R. Todd Stravitz, MD: Planning/conducting study, Analysis of the data, Drafting manuscript.

\section{ORCID}

Puneet Puri (iD https://orcid.org/0000-0001-8953-345X

William M. Lee iD https://orcid.org/0000-0002-2783-5441

\section{REFERENCES}

1. Reuben A, Tillman H, Fontana RJ, et al. Outcomes in adults with acute liver failure between 1998 and 2013: an observational cohort study. Ann Intern Med. 2016;164(11):724-732.

2. Bernal W, Wendon J. Acute liver failure. $N$ Engl J Med. 2013;369(26):2525-2534

3. Zimmerman HJ, Maddrey WC. Acetaminophen (paracetamol) hepatotoxicity with regular intake of alcohol: analysis of instances of therapeutic misadventure. Hepatology. 1995;22(3):767-773.

4. Wolf KK, Wood SG, Allard JL, et al. Role of CYP3A and CYP2E1 in alcohol-mediated increases in acetaminophen hepatotoxicity: comparison of wild-type and Cyp2e1(-/-) mice. Drug Metab Dispos. 2007;35(7):1223-1231.

5. Mitchell JR, Jollow DJ, Potter WZ, et al. Acetaminophen-induced hepatic necrosis. I. Role of drug metabolism. J Pharmacol Exp Ther. 1973;187(1):185-194.

6. Mitchell JR, Thorgeirsson SS, Potter WZ, Jollow DJ, Keiser H. Acetaminophen-induced hepatic injury: protective role of glutathione in man and rationale for therapy. Clin Pharmacol Ther. 1974;16(4):676-684.

7. Lauterburg BH, Velez ME. Glutathione deficiency in alcoholics: risk factor for paracetamol hepatotoxicity. Gut. 1988;29(9):1153-1157.

8. Wong LT, Whitehouse LW, Solomonraj G, Paul CJ. Effect of a concomitant single dose of ethanol on the hepatotoxicity and metabolism of acetaminophen in mice. Toxicology. 1980;17(3):297-309.

9. Schmidt LE, Dalhoff K, Poulsen HE. Acute versus chronic alcohol consumption in acetaminophen-induced hepatotoxicity. Hepatology. 2002;35(4):876-882.

10. Koch DG, Speiser JL, Durkalski V, et al. The natural history of severe acute liver injury. Am J Gastroenterol. 2017;112(9):1389-1396.

11. Read RB, Tredger JM, Williams R. Analysis of factors responsible for continuing mortality after paracetamol overdose. Hum Toxicol. 1986;5(3):201-206.

12. Makin AJ, Wendon J, Williams R. A 7-year experience of severe acetaminophen-induced hepatotoxicity (1987-1993). Gastroenterology. 1995;109(6):1907-1916.

13. Larson AM, Polson J, Fontana RJ, et al. Acetaminophen-induced acute liver failure: results of a United States multicenter, prospective study. Hepatology. 2005;42(6):1364-1372.

14. Prescott LF. Paracetamol, alcohol and the liver. Br J Clin Pharmacol. 2000;49(4):291-301.

15. Thummel KE, Slattery JT, Ro $\mathrm{H}$, et al. Ethanol and production of the hepatotoxic metabolite of acetaminophen in healthy adults. Clin Pharmacol Ther. 2000;67(6):591-599.

16. Thummel KE, Slattery JT, Nelson SD. Mechanism by which ethanol diminishes the hepatotoxicity of acetaminophen. J Pharmacol Exp Ther. 1988;245(1):129-136.

17. O'Grady JG, Alexander GJ, Hayllar KM, Williams R. Early indicators of prognosis in fulminant hepatic failure. Gastroenterology. 1989;97(2):439-445.

18. Schmidt LE. Age and paracetamol self-poisoning. Gut. 2005;54(5):686-690.

19. Myers R, Shaheen A, Li B, Dean S, Quan H. Impact of liver disease, alcohol abuse, and unintentional ingestions on the outcomes of acetaminophen overdose. Clin Gastroenterol Hepatol. 2008;6(8):918-925.

20. Mitchell MC, Schenker S, Speeg KV Jr. Selective inhibition of acetaminophen oxidation and toxicity by cimetidine and other histamine $\mathrm{H} 2$-receptor antagonists in vivo and in vitro in the rat and in man. J Clin Invest. 1984;73(2):383-391.

21. Suzuki A, Yuen N, Walsh J, Papay J, Hunt CM, Diehl AM. Comedications that modulate liver injury and repair influence clinical outcome of acetaminophen-associated liver injury. Clin Gastroenterol Hepatol. 2009;7(8):882-888. 
22. Rutherford A, Davern T, Hay JE, et al. Influence of high body mass index on outcome in acute liver failure. Clin Gastroenterol Hepatol. 2006;4(12):1544-1549.

23. Holt EW, DeMartini S, Davern TJ. Acute liver failure due to acetaminophen poisoning in patients with prior weight loss surgery: a case series. J Clin Gastroenterol. 2015;49(9):790-793.

\section{SUPPORTING INFORMATION}

Additional supporting information may be found online in the Supporting Information section.
How to cite this article: Puri P, Lee WM, Fontana RJ, et al. Alcohol consumption is associated with the severity and outcome of acute liver injury/failure. Liver Int. 2020;40:360367. https://doi.org/10.1111/liv.14327 